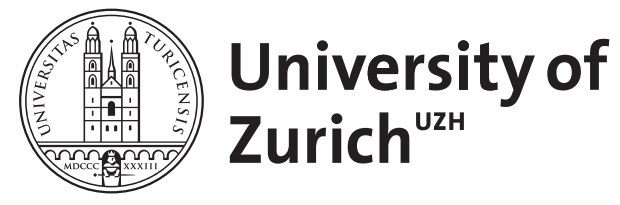

Zurich Open Repository and Archive

University of Zurich

University Library

Strickhofstrasse 39

CH-8057 Zurich

www.zora.uzh.ch

Year: 2006

\title{
Kann Philosophie zum Dialog der Religionen beitragen? Anmerkungen zur Koranexegese des Nikolaus von Kues
}

\author{
Rudolph, Ulrich
}

Posted at the Zurich Open Repository and Archive, University of Zurich

ZORA URL: https://doi.org/10.5167/uzh-80133

Book Section

Published Version

Originally published at:

Rudolph, Ulrich (2006). Kann Philosophie zum Dialog der Religionen beitragen? Anmerkungen zur Koranexegese des Nikolaus von Kues. In: Speer, Andreas; Wegener, Lydia. Wissen über Grenzen : arabisches Wissen und lateinisches Mittelalter. Berlin: de Gruyter, 179-193. 


\title{
Kann Philosophie zum Dialog der Religionen beitragen? Anmerkungen zur Koranexegese des Nikolaus von Kues
}

\author{
UlRICH RUdOLPH (Zürich)
}

Der Fall von Konstantinopel im Jahr 1453 hat die lateinische Christenheit tief erschüttert. Obwohl das Ereignis vorhersehbar war, weil sich die Osmanen längst auf dem Balkan festgesetzt hatten, war man doch konsterniert, als es tatsächlich eintrat. Entsprechend heftig klangen die Reaktionen, die damals in Europa geäußert wurden. Sie fielen, wie es bei dramatischen Ereignissen meistens geschieht, höchst unterschiedlich aus. Einige Akteure meinten, man solle sofort einen Kreuzzug ausrufen und den Heiden auf dem Schlachtfeld entgegentreten. Andere dachten eher an eine literarische Offensive; sie setzten ihre Hoffnungen auf eine umfassende polemische Schrift, in der sämtliche Irrlehren der Muslime ein für alle Mal entlarvt werden sollten. Daneben gab es aber auch einige wenige Personen, die nicht martialisch, sondern besonnen reagierten. Sie wollten jeden unnötigen Konflikt vermeiden. Deswegen plädierten sie dafür, eine Konferenz einzuberufen. Auf ihr sollten sich Vertreter der verschiedenen Religionen zusammensetzen und über die Wahrheit(en) ihres Glaubens diskutie$\operatorname{ren}^{1}$.

Keiner dieser Vorschläge ist letztlich in die Tat umgesetzt worden. Statt dessen gewann der politische Pragmatismus die Oberhand. Nach einiger Zeit wurde das osmanische Reich in die bestehenden diplomatischen Beziehungen eingebunden und als das, was es war: eine Großmacht mit umfangreichen europäischen Besitzungen, anerkannt. Trotzdem ist die Debatte, die in den Jahren nach 1453 stattfand, für uns von großem Interesse. Denn sie besaß eine Intensität

\footnotetext{
1 Über die verschiedenen Reaktionen in Europa orientieren A. Pertusi, La caduta di Constantinopoli. Le testimonianze dei contemporanei, Mailand 1976, und E. Meuthen, Der Fall von Konstantinopel und der lateinische Westen, in: Mitteilungen und Forschungsbeiträge der CusanusGesellschaft (= MFCG) 16 (1984), 15-30. Die Idee, eine Konferenz abzuhalten, wurde vor allem von Johannes von Segovia propagiert, der in einer umfangreichen Korrespondenz versuchte, wichtige Repräsentanten der Kirche für diesen Vorschlag zu gewinnen. Cf. dazu R. Haubst, Johannes von Segovia im Gespräch mit Nikolaus von Kues und Jean Germain über die göttliche Dreieinigkeit und ihre Verkündigung vor den Mohammedanern, in: Münchener Theologische Zeitschrift 2 (1951), 115-129; D. Cabanelas Rodriguez, Juan de Segovia y el problemo islámico, Madrid 1952; W. Heinemann, Einheit in Verschiedenheit. Das Konzept eines intellektuellen Religionenfriedens in der Schrift ,De pace fidei' des Nikolaus von Kues, Altenberge 1987, 27 sqq.
} 
und ein Niveau, die in Diskussionen über den Islam - in den Jahrhunderten zuvor und danach - selten erreicht worden sind. Maßgeblichen Anteil daran hatte Nicolaus Cusanus. Seine Beiträge zum Thema waren originell und entwarfen eine ungewöhnliche Perspektive, über deren Tragweite bis heute nachgedacht wird ${ }^{2}$.

I.

Daß der Cusaner an der Debatte teilnahm, hatte mehrere Gründe. Zum einen interessierte ihn das Thema. Spätestens seit er im Jahre 1437 als Mitglied einer päpstlichen Delegation an den Bosporus gereist war, beschäftigte er sich mit den islamischen Lehren und besorgte sich Bücher, in denen er Auskünfte über sie erhielt ${ }^{3}$. Zum anderen hatte Nikolaus von Kues eine öffentliche Funktion. Er bekleidete - zunächst in Brixen, später in Rom - hohe kirchliche Ämter. Infolgedessen war er auch von Berufs wegen dazu gezwungen, in dieser Frage, die allenthalben die Gemüter bewegte, Stellung zu beziehen ${ }^{4}$.

All diese Motive und Hintergründe haben Spuren in seinem Wirken hinterlassen. Denn die Vorgehensweise, die er nach 1453 wählte, war keineswegs eindeu-

2 Die ältere Literatur zu Cusanus' Auseinandersetzung mit dem Islam ist zusammengestellt in meinem Aufsatz: Nikolaus von Kues und der Dialog mit dem Islam, in: Der Islam 71 (1994), 95-108, hier: 98, nt. 8. Seither sind zahlreiche weitere Beiträge zum Thema erschienen, darunter J. Hafner, Welche Geschäftsordnung braucht das Religionsgespräch? Zur Konstruktion idealer Dialoge bei Lessing, Lullus und Cusanus, in: K. Kienzler/G. Riedl/M. Schiefer-Ferrari (eds.), Islam und Christentum. Religion im Gespräch (Augsburger Schriften zur Theologie und Philosophie 1), Münster 2001, 171-188; W. A. Euler, Cusanus’ Verständnis der negativen Theologie und seine Auseinandersetzung mit nichtchristlichen Religionen in De pace fidei und Cribratio Alkorani, in: Theologische Quartalschrift 181 (2001), 132-142; id., Gewohnheit ist kein Attribut Gottes: Die Intention des Religionsdialoges bei Abélard, Lull und Cusanus, in: K. Yamaki (ed.), Nicholas of Cusa: A Medieval Thinker for the Modern Age, Richmond 2002, 153-166; id., Nikolaus von Kues als Wegbereiter des interreligiösen Dialogs und der Theologie der Religionen, in: MFCG 28 (2003), 211-231; M. Riedenauer, Logik, Rationalität und religiöse Rede nach Nikolaus Cusanus, in: M. Lutz-Bachmann/A. Fidora (eds.), Juden, Christen und Muslime. Religionsdialoge im Mittelalter, Darmstadt 2004, 192-220; H. Schrödter, Religion zwischen Diskurs und Gewalt: Diskurstheoretische Elemente bei Nikolaus von Kues - Anfragen an die Diskurstheorie, in: ibid., 221-238; W. Knoch, Verteidigung als Annäherung? Die Auseinandersetzung des Nikolaus von Kues mit dem Islam im Spiegel der, Cribratio Alkorani‘, in: J. A. Aertsen/M. Pickavé (eds.), ,Herbst des Mittelalters? Fragen zur Bewertung des 14. und 15. Jahrhunderts (Miscellanea Mediaevalia 31), Berlin-New York 2004, 393-405.

3 Der Anlaß für die Reise nach Konstantinopel waren die Unionsverhandlungen mit der oströmischen Kirche; zum historischen Hintergrund cf. W. Krämer, Der Beitrag des Nikolaus von Kues zum Unionskonzil mit der Ostkirche, in: MFCG 9 (1971), 34-52. Daß Cusanus bei dieser Gelegenheit versuchte, Informationen über den Islam zu erhalten, schreibt er selbst im ersten Prolog zu seiner, Cribratio Alkorani‘. Dort gibt er auch an, daß er sich immer wieder um Bücher über die islamischen Glaubenslehren bemüht habe (h VIII, nn. 2-3; zur Edition der ,Cribratio“ siehe unten, nt. 11).

4 Zum Lebensweg und den verschiedenen kirchlichen Ämtern, die Cusanus innehatte, cf. E. Meuthen, Nikolaus von Kues. Skizze einer Biographie, Münster 1964, ${ }^{5} 1982$. 
tig, sondern läßt den Schluß auf mehrere Interessenlagen und Rücksichtnahmen zu. Als päpstlicher Legat befolgte Cusanus die Anweisung, die Kreuzzugsvorbereitungen zu koordinieren ${ }^{5}$. Als Kirchenlehrer beauftragte er einen Schüler damit, er möge die islamischen Lehren in einer Polemik widerlegen ${ }^{6}$. Sein eigentliches Anliegen dürfte indessen die dritte Option gewesen sein, also der Dialog mit den Muslimen. Diese Idee trat nämlich immer dann in den Vordergrund, wenn Cusanus selbst zur Feder griff, um seine Gedanken zu entwickeln - sei es in Briefen an Freunde oder in Schriften, die für die Öffentlichkeit bestimmt waren.

Zum ersten Mal geschah das im Sommer 1453, also unmittelbar nach dem Fall von Konstantinopel. In dieser Zeit entstand bereits sein erster wichtiger Text zum Thema, der Dialog, De pace fidei ${ }^{6}$ (Über den Frieden im Glauben ${ }^{6}{ }^{7}$. In diesem Werk wird eine fiktive Konferenz geschildert, die unter dem Vorsitz Gottes im Himmel stattfindet. Siebzehn Vertreter verschiedener Völker und Glaubensgemeinschaften sind zusammengekommen, um nacheinander mit dem göttlichen Wort und mit den Aposteln Petrus und Paulus über die Grundwahrheiten der Religion(en) zu diskutieren. Der Kreis der Gäste ist weit gespannt. Er reicht von einem Inder über einen Griechen und einen Böhmen bis zu einem Spanier. Entsprechend breit ist das Spektrum der theologischen Fragen, die diskutiert werden, angelegt. Trotzdem fällt auf, daß der Islam in der Debatte einen herausragenden Platz einnimmt. Denn seine Lehren werden nicht nur von drei Teilnehmern - einem Araber, einem Perser und einem Türken - explizit vertreten. Sie kommen auch in verschiedenen anderen Beiträgen zur Sprache, so etwa, wenn der Delegierte aus Deutschland den Ratschlag erhält, er möge doch bitte, wenn er den Islam richtig verstehen wolle, Avicennas Bücher lesen ${ }^{8}$.

Das Szenario mutet ausgesprochen modern an; es erinnert an Konferenzen unserer Tage. Deswegen ist es auch nicht verwunderlich, wenn Cusanus' himmlische Versammlung vor kurzem mit einem Gipfeltreffen der Europäischen Gemeinschaft verglichen wurde? ${ }^{9}$ Einen Unterschied gibt es jedoch, und er ist

\footnotetext{
5 Das geschah vor allem in seinen letzten Lebensjahren, als Papst Pius II. den Kreuzzugsgedanken energisch propagierte; dazu E. Meuthen, Die letzten Jahre des Nikolaus von Kues. Biographische Untersuchungen nach neuen Quellen, Köln-Opladen 1958.

6 So entstand die Schrift ,Contra perfidiam Mahometi' des Dionysius Cartusianus; dazu L. Hagemann, Der Kur'ān in Verständnis und Kritik bei Nikolaus von Kues. Ein Beitrag zur Erhellung islamisch-christlicher Geschichte (Frankfurter Theologische Studien 21), Frankfurt a. M. 1976, 50 sqq.

7 Kritische Edition von R. Klibansky/H. Bascour, Nicolai de Cusa Opera omnia iussu et auctoritate Academiae Litterarum Heidelbergensis ad codicum fidem edita. VII: De pace fidei cum epistula ad Ioannem de Segobia, Hamburg 1959, ${ }^{2} 1970$ (= h VII). Lat.-dt. Lesetext von K. Berger u. Ch. Nord u. d. T. Nikolaus von Kues. Vom Frieden zwischen den Religionen, Frankfurt a. M.-Leipzig 2002. Zum Werk cf. Heinemann, Einheit (nt. 1); W. A. Euler, Unitas et Pax. Religionsvergleich bei Raimundus Lullus und Nikolaus von Kues, Würzburg-Altenberge $1990,{ }^{2} 1995$.

8 Cf. h VII, n. 52 (49, 16-19).

9 Cf. Euler, Nikolaus von Kues als Wegbereiter (nt. 2), 211 sqq.
} 
letztlich entscheidend. Denn Cusanus kann am Ende seiner Konferenz ein überzeugendes Ergebnis vorweisen. Es besagt, daß sämtliche Religionen in den fundamentalen Fragen, d. h. in ihren Annahmen über Gott und die Menschen, übereinstimmen. Wenn trotzdem große Unterschiede zwischen den einzelnen Glaubensgemeinschaften feststellbar seien, liege das nur an den rituellen und kultischen Details. Man könne deswegen getrost von einer Harmonie zwischen den Glaubensgemeinschaften ausgehen. Sie geht laut Cusanus so weit, daß er dafür die berühmte Formel „eine Religion in verschiedenen Riten“ („una religio in rituum varietate") verwendet hat ${ }^{10}$.

,De pace fidei“ enthielt also eine Vision, und allein das ist angesichts der Entstehungszeit des Werkes erstaunlich. Denn die Vision schloß den erklärten Feind jener Tage, die arabischen, persischen und vor allem türkischen Muslime, bewußt mit ein. Trotzdem war die Aufgabe, der sich Cusanus verschrieben hatte, noch nicht erfüllt. Denn der Text entwarf lediglich ein Programm, er nannte für seine weitreichenden Thesen kaum Argumente. Sie mußten erst noch erbracht werden, insbesondere im Falle des Islams, der in den Augen vieler Christen nach wie vor als ein Werk des Antichristen galt.

Das geschah in der zweiten Schrift, die Nikolaus von Kues unserem Thema widmete. Gemeint ist die ,Cribratio Alkorani' (,Sichtung des Korans'), die im Winter 1460/61 entstand ${ }^{11}$. Sie diente einem konkreten Zweck, nämlich mögliche Verhandlungen mit den Muslimen vorzubereiten. Deswegen hat der Text auch einen anderen Charakter: Er beschreibt keine visionären Entwürfe, sondern gibt dem Papst Argumente an die Hand. Sie gehen wieder von der Annahme aus, daß zwischen den beiden Religionen eine große Nähe bestehe. Insofern ist das Konzept der Harmonie, das in ,De pace fidei ${ }^{6}$ entwickelt wurde, auch in der ,Cribratio“ vorausgesetzt. Gleichzeitig konkretisiert Cusanus aber seine alte Idee und gibt ihr dabei einen neuen Akzent. Denn jetzt wird deutlicher als vorher, in welchem Sinn er die Übereinstimmung zwischen Christentum und Islam postuliert. Sie soll nicht etwa darin bestehen, daß beide Religionen als gleichberechtigte Ausdrucksformen derselben Wahrheit gelten. Vielmehr sieht Cusanus zwischen ihnen eine klare und unumstößliche Hierarchie: Das Christentum ist sein Maßstab. Es repräsentiert die Wahrheit in ihrer reinen und vollkommenen Form, denn es wurde von Jesus Christus, dem Sohn Gottes, begründet.

10 h VII, n. 6 (7, 10-11): „, [...] et cognoscent omnes quomodo non est nisi religio una in rituum varietate." Die Originalität dieser Formel wird allerdings oft überschätzt. Wie Ch. Auffarth, Das Ende des Pluralismus, in: id. (ed.), Religiöser Pluralismus im Mittelalter?, Münster 2006 (im Druck) zeigt, nimmt sie eine Idee auf, die bereits lange (viertes Laterankonzil 1215) als Modell für eine unierte und universale Kirche diskutiert wurde. Das erklärt, warum Cusanus dieselbe Formel auch bei seiner Auseinandersetzung mit den Hussiten verwendet hat; dazu E. Meuthen, Der ,Dialogus concludens Amedistarum errorem ex gestis et doctrina concilii Basiliensis', in: MFCG 8 (1970), 58-62, und H. Hallauer, Das Glaubensgespräch mit den Hussiten, in: MFCG 9 (1971), 53-75.

11 Kritische Edition von L. Hagemann, Nicolai de Cusa Opera omnia. VIII: Cribratio Alkorani, Hamburg 1986 (= h VIII). Lat.-dt. Textausgabe von L. Hagemann/R. Glei u. d. T. Nicolai de Cusa, Cribratio Alkorani. Sichtung des Korans, 3 vols. (Philosophische Bibliothek 420 a-c), 
Der Islam erfüllt diese Bedingung dagegen nicht; also kann er auch keinen direkten Zugang zur Wahrheit für sich in Anspruch nehmen. Seine Aussagen können nur dann wahr sein, wenn sie mit den Lehren des Christentums übereinstimmen $^{12}$.

Das klingt höchst restriktiv, aber Cusanus meint, auf diese Weise das Gespräch mit den Muslimen eröffnen zu können. Der Grund dafür ist seine Überzeugung, daß die Übereinstimmungen zwischen Islam und Christentum in der Tat zahlreich seien. Das zu demonstrieren, ist die Aufgabe der ,Cribratio'. In ihr wird der heilige Text der Muslime also auf seine christlichen Reminiszenzen hin untersucht. Wie zu erwarten war, mit Erfolg. Denn als Resultat seiner Bemühungen kann Cusanus festhalten, daß alle zentralen christlichen Lehren im Koran erwähnt und anerkannt werden: die Trinitätslehre ${ }^{13}$, der Kreuzestod ${ }^{14}$, die Auferstehung $^{15}$, die Erlösung durch Christus ${ }^{16}$, ja selbst die Gottessohnschaft Jesu ${ }^{17}$, die bekanntlich der größte Zankapfel zwischen den beiden Glaubensgemeinschaften gewesen ist. All das gelingt Cusanus natürlich nur, weil er eine eigenwillige Form der Koranexegese anwendet. Er fragt nie danach, wie die Muslime selbst ihren heiligen Text verstehen, sondern deutet ihn einfach aus der christlichen Perspektive und nimmt überdies eine ganze Reihe von sachlichen Irrtümern und Mißverständnissen in Kauf. Gleichwohl ist der Kardinal davon überzeugt, der Schrift keine Gewalt anzutun. Denn er glaubt ja, mit seinem Vorgehen den Muslimen einen Zugang zur Wahrheit zu eröffnen. Statt sie zu bekämpfen, möchte er sie an die Hand nehmen (manuductio) ${ }^{18}$ und ihnen durch seine ,fromme Auslegung' (pia interpretatio $)^{19}$ zeigen, welche geistigen Schätze im Koran verborgen sind.

Auch dieser Ansatz ist in der Sekundärliteratur mit einem gewissen Wohlwollen aufgenommen worden. Das geschah allerdings nicht so euphorisch, wie es bei den Überlegungen von ,De pace fidei‘ der Fall war. Auffällig ist eher, daß die zahlreichen Interpreten, die sich inzwischen mit dem Text beschäftigt haben, jeweils einen anderen Aspekt in den Vordergrund ihrer Bewertungen gerückt haben. Manche betonen, daß Cusanus auch in der ,Cribratio' an der Idee des

Hamburg 1989-1993. Zum Werk cf. Hagemann, Der Kur'ān (nt. 6); id., Nikolaus von Kues im Gespräch mit dem Islam (Christlich-Islamisches Institut; Abhandlungen 1), Altenberge 1983.

12 h VIII, n. 41, 1-3: „Hinc si quid pulchri, veri et clari in Alkorano reperitur, necesse est, quod sit radius lucidissimi evangelii; et hoc verum videt, qui evangelio lecto ad Alkoranum se transfert."

13 Cf. h VIII, nn. 99-114.

14 Cf. h VIII, nn. 115-124.

15 Cf. h VIII, nn. 125-132.

16 Cf. h VIII, nn. 210 u. $229-234$.

17 Cf. h VIII, nn. $54-74$.

18 So Cusanus' eigene Formulierung, etwa in den Titeln zu h VIII, n. 99 („Manuductio ex his quae in mundo sunt, ut videatur deus trinus"), n. 101 („Manuductio de intellectuali trinitate ad divinam") und n. 103 („Manuductio eiusdem per amorem“). Zum methodischen Prinzip ausführlich Hagemann, Der Kur'ān (nt. 6), 72 sq. u. 162-172.

19 h VIII, n. 86, 3-6: „Nunc accedamus ad elucidationem trinitatis, quam in divinitate colimus, et ostendamus Alkoranum pia interpretatione non contradicere trinitati, modo quo nos de ipsa loquimur, qui evangelio inhaeremus“; cf. h VIII, n. 119, 1; n. 124, 3 sq.; n. 154, 8. Zum Prinzip Hagemann, Der Kur’ān (nt. 6), 72. 
Dialogs festhält ${ }^{20}$. Andere bemängeln seinen interpretatorischen Parforceritt ${ }^{21}$. Wieder andere weisen darauf hin, daß dieser zweite Text - im Gegensatz zur Friedensschrift - zahlreiche polemische Töne enthalte, übrigens nicht nur gegenüber den Muslimen, sondern auch gegenüber den Juden, die mehrmals heftig attackiert werden ${ }^{22}$.

20 Großen Beifall fand der Text beispielsweise auf dem Symposion ,Nikolaus von Kues als Promotor der Ökumene، (Bernkastel-Kues, 22.-24. September 1970), dessen Ergebnisse in den MFCG 9 (1971) veröffentlicht worden sind. Dort erklärt A. Schall (87): „Es wird immer noch behauptet, das Mittelalter hätte sich nur in einem wilden, bösen Kampf gegen den Islam betätigt. $\mathrm{Daß}$ eine so positive Stellungnahme vorgelegen hat wie in der Cribratio Alchoran, ist offensichtlich bisher kaum durchgedrungen." R. Haubst meint an gleicher Stelle (87, nt. 17), Cusanus habe einen ,Brückenschlag“ zum Islam gesucht, und B. Hanssler attestiert dem Kardinal gar (190), „,der kühnste aller Ökumeniker und [...] der engagierte Anwalt der Völkerverständigung“ gewesen zu sein. In der neueren Forschung wird die ,Cribratio“ zurückhaltender beurteilt, aber auch hier begegnen uns zahlreiche positive Stimmen. So äußert Euler, Nikolaus von Kues als Wegbereiter (nt. 2), 228: „Es sind diese Nuancen der cusanischen Religionsspekulation, die es rechtfertigen, Nikolaus von Kues als einen Wegbereiter des interreligiösen Dialoges und zugleich einer die Begegnung zwischen den Religionen systematisch begründenden und fördernden Theologie der Religionen zu sehen.“ Und Knoch, Verteidigung (nt. 2), 403, stellt fest: „Dennoch, bei aller Verwurzelung in der apologetischen Tradition, gelangt Cusanus in seinem speziellen Vorgehen zu einer Annäherung an den Gegner, wie sie vorher unbekannt war [...]. Dem Koran wird echtes Eigengewicht zugemessen, wenn auch mit Hilfe von Verzeichnungen, und damit ist ein entscheidender Schritt von der reinen Polemik hin zum sachorientierten Dialog getan."

21 Hagemann, der sich am ausführlichsten mit der ,Cribratio‘ befaßt hat, erkennt die Dialogbereitschaft des Cusaners an, wenn er in der Einleitung zu Der Kur'ān (nt. 6), 3, schreibt: ,,[...] ist es doch Nikolaus von Kues, dem in der Geschichte des Ökumenismus oder dessen Vorbereitung zweifellos der erste Platz zukommt.“ Gleichwohl bilanziert er am Ende seiner Untersuchung (ibid., 181): „Cusanus hat versucht, nicht nur jene genuin biblischen Elemente gleichsam mäeutisch aus dem Kur'ān zu eruieren, sondern er hat darüber hinaus vom theologischen Fundament des christlichen Glaubens aus spezifisch christliche Glaubensinhalte in den Kur'ān hineinzuinterpretieren versucht. Darin liegt die Problematik seiner Kur’āndeutung.“ Noch kritischer klingt das Fazit in seinem später erschienenen Beitrag ,Nikolaus von Kues im Gespräch“ (nt. 11), 26 sq. Wie problematisch die Interpretationsansätze in der ,Cribratio' sind, hatte im übrigen schon G. C. Anawati, Nicolas de Cues et le problème de l'Islam, in: Nicolò Cusano agli inizi del mondo moderno. Atti del Congresso internazionale in occasione del V centenario della morte di Nicolò Cusano, Bressanone, 6-10 settembre 1964, Florenz 1970 (Pubblicazioni della Facoltà di Magistero dell'Università di Padova 12), 141 -173, deutlich gemacht. Er resümierte seine Überlegungen (172) mit den später häufig zitierten Worten: „Nicolas de Cues peut-il nous être utile pour le dialogue islamo-chrétien? Au risque de déplaire aux admirateurs sans réserve du célèbre Cardinal, je répondrai sans hésitation: Non. Nous n'aimerions certainement pas qu'un livre comme la Cribratio Alchorani tombât entre les mains de nos amis musulmans: ils en seraient littéralement scandalisés. Il faudrait leur expliquer avec force de détails que Nicolas de Cues représente un progrès sur ses prédécesseurs, qu'on ne trouve pas chez lui des torrents d'injures à l'égard de Mahomet etc. Il n'est pas sûr qu'ils comprendraient. Mieux vaut ne pas tenter l'aventure.“

$22 \mathrm{Daß}$ die ,Cribratio' dezidiert antijüdische Äußerungen enthält, betont vor allem K. Flasch, Nikolaus von Kues. Geschichte einer Entwicklung. Vorlesungen zur Einführung in seine Philosophie, Frankfurt a. M. 1998. Sie stehen, wie er anhand der ,Acta Cusana' nachweist (350 sq.), in einer Linie mit anderen antijüdischen Behauptungen und Maßnahmen des Cusaners. Deswegen vermutet Flasch zu Recht, daß manche islamkritische Aussage in der, Cribratio‘ eigentlich auf die Juden zielte (545): „Sozialpsychologisch ist dieser Griff der mittelalterlichen Polemiker, den auch 
Diesen verschiedenen Urteilen möchte ich kein weiteres hinzufügen. Es könnte ohnehin keine neue Gesamtsicht auf den Text bieten, sondern nur eine modifizierte Gewichtung der bekannten Beobachtungen sein. Statt dessen möchte ich mich im Folgenden auf einen einzelnen Aspekt beschränken. Gemeint ist die Frage, ob und inwiefern die Philosophie bei Cusanus' Überlegungen zum Islam eine Rolle gespielt hat. Auch dieser Gesichtspunkt ist in verschiedenen Forschungsbeiträgen schon zur Sprache gekommen ${ }^{23}$. Das geschah in der Regel mit einem positiven Ergebnis. Man konstatierte, seine philosophische Grundhaltung habe dazu beigetragen, daß er sich fremden Vorstellungen öffnete und zu einem Dialog bereit war ${ }^{24}$. Diese Einschätzung soll im Folgenden noch einmal am Beispiel des Islams überprüft werden. Das geschieht in zwei Schritten, die sich dem Problem jeweils von einer anderen Seite aus nähern. Zunächst wird gefragt, wie Cusanus mit dem Spezifikum der islamischen Religion umging, d. h. wie er den Anspruch, Mohammed sei ein Prophet gewesen, einschätzte. Anschließend kommt eine allgemeinere Perspektive zur Sprache. Dann geht es um die Frage, von welchen anthropologischen und theologischen Prämissen Cusanus ausging, als er sich auf die Beschäftigung mit dem Islam einließ.

\section{II.}

Beginnen wir also mit Mohammed. Sein Auftreten wird im Verlauf der ,Cribratio' mehrfach geschildert. Zum ersten Mal geschieht das im Vorwort. Dort geht es Cusanus zwar darum, seine grundsätzliche Auffassung von Religion darzulegen; aber dabei kommt er schnell auf sein konkretes Thema, den Begründer der islamischen Glaubensgemeinschaft, zu sprechen. In diesem Zusammenhang heißt es ${ }^{25}$, alle Menschen trügen in sich ein Streben nach Gott, dem Einen und

Cusanus nicht ausläßt, bedenkenswert: Sie bekämpfen den fernlebenden Feind so, daß auch die nahewohnenden Gegner einen Schlag abbekommen. Es springt dabei etwas ab für den westlichen Hausgebrauch: Die Juden - auch Cusanus spricht von perversi Iudaei als Werkzeugen des Teufels - sind mit schuld, daß die Türken so schlimm sind.“

23 Cf. F. H. Burgevin, Cribratio Alchorani. Nicholas Cusanus' Criticism of the Koran in the Light of His Philosophy of Religion, New York e. a. 1969; K. Kremer, Die Hinführung (manuductio) von Polytheisten zum Einen, von Juden und Muslimen zum Dreieinen Gott, in: MFCG 16 (1984), 126-163; Flasch, Nikolaus von Kues (nt. 22), 330-382 (zu ,De pace fidei') u. $541-$ 553 (zur, Cribratio').

24 So meint beispielsweise Kremer, Die Hinführung (nt. 23), 158: „Cusanus hat sich aufgemacht, [...] um die jedem Glauben gemeinsam zugrunde liegenden Voraussetzungen aufzudecken. Dafür bietet er den ganzen Genius seiner spekulativen Befähigung auf [...].“ Ähnliche Annahmen finden sich im übrigen auch in Beiträgen, die sich nicht ausdrücklich mit dem philosophischen Hintergrund der cusanischen Religionsbetrachtungen beschäftigen, etwa bei Knoch, Verteidigung (nt. 2), 403: „Gegen den Erfolg der islamischen (türkischen) Expansionspolitik stellte Cusanus eine Auseinandersetzung mit den allen Religionen zugänglichen Waffen des philosophischen Denkens, um die allgemeine Einsichtigkeit der christlichen Grunddogmen und so die Überlegenheit der christlichen Religion zur Geltung zu bringen."

25 Im Folgenden wird der Inhalt von h VIII, nn. 5, 1-9, 5 zusammengefaßt. 
höchsten Guten. Aufgrund ihrer menschlichen Begrenztheit sei ihnen jedoch die Erkenntnis der göttlichen Wahrheit versperrt. Deswegen seien im Lauf der Geschichte mehrere Weise und Propheten aufgetreten, um uns den Weg zum Heil zu zeigen. An ihrer Spitze stünden Moses, Jesus und Mohammed, denen wir „die namhafteren Beschreibungen des besagten Weges“ („magis famosae descriptiones dictae viae $\left.{ }^{\text {" }}\right)^{26}$ zu verdanken hätten. Nur Jesus könne indessen die Wahrheit tatsächlich gewußt haben. Denn er war nicht nur ein Mensch, sondern gleichzeitig „die allwissende göttliche Weisheit" (,omnisciens divina sapientia“) ${ }^{27}$. Moses und Mohammed seien dagegen bloße Geschöpfe gewesen - und damit wie alle Menschen außerstande, die göttliche Wahrheit in ihrer Fülle zu erkennen ${ }^{28}$.

Die Überlegung ist interessant und verdient eigentlich eine genauere Betrachtung. Für uns ist im Moment aber nur von Bedeutung, wie an dieser Stelle Mohammed beurteilt wird. Dazu erfahren wir zweierlei. Erstens: Mohammed war ein Prophet; Cusanus ist bereit, diesen Anspruch anzuerkennen. Er stellt ihn auf eine Stufe mit Moses und gesteht beiden zu, herausragende Weise und Wahrheitssucher gewesen zu sein. Zweitens: Trotz dieser Leistung waren Mohammed und Moses nur Menschen. Sie konnten die Wahrheit gar nicht aus eigener Kraft erfassen. Also können die Religionen, die von ihnen begründet wurden, auch nicht dem Christentum, das auf Gott selbst zurückgeht, gleichgestellt sein.

Schon das klingt mehrdeutig, aber Cusanus' Auseinandersetzung mit der Person Mohammeds geht weiter. Das zeigt sich bereits wenige Seiten später, wenn das Thema zum zweiten Mal aufgegriffen und mit einer anderen Gewichtung behandelt wird. Jetzt werden uns nämlich Einzelheiten aus dem Leben des Propheten berichtet. Dabei wird deutlich, daß Cusanus keinen eigenen Zugang zu dessen Biographie besitzt, sondern weitgehend den älteren lateinischen Darstellungen verpflichtet ist. Mohammed, so hören wir nun, sei in Mekka von einem Mönch namens Sergius unterrichtet worden. Dieser habe ihm das Evangelium in der nestorianischen Form gepredigt, und der gelehrige Schüler sei der neuen Botschaft auch anfangs mit großem Eifer gefolgt. Indessen: Drei äußerst listige Juden (,tres astutissimi Iudaei ${ }^{\circ}$ ) hätten es verstanden, ihn wieder vom wahren Glauben abzubringen. Sie schlossen sich ihm an und redeten ihm allerlei üble Dinge ein - ,ne perfectus fieret", „damit er nicht vollkommen werde“. Doch damit nicht genug: Selbst als Mohammed gestorben war, hörten sie nicht auf, gegen ihn und seine Anhänger zu intrigieren. Denn jetzt machten sie sich an 'Alī ibn abī Ṭālib heran, ließen sich von ihm den Koran aushändigen und „machten nach eigenem Gutdünken teils Zusätze, teils Abstriche in Mohammeds Buch“ (,et apposuerunt et deposuerunt de libro Mabumeti, quae voluerunt" ${ }^{29}$.

26 h VIII, n. 7, 14

27 h VIII, n. 8, 16.

28 Cf. h VIII, n. 8, 4-10.

29 h VIII, n. $11,1-14$. 
Hier ist sie also, die oben angekündigte, scharfe Polemik. Sie blitzt in der ,Cribratio' immer wieder auf, wobei, wie gesagt, nicht die Muslime, sondern die Juden die privilegierte Zielscheibe sind. Gleichwohl haben solche Äußerungen Rückwirkungen auf das Bild des islamischen Propheten. Er gilt jetzt nicht mehr als der aufrechte Wahrheitssucher. Nun wird ihm vielmehr vorgeworfen, daß er die Wahrheit - dank der Begegnung mit dem christlichen Mönch - berühren durfte und doch - unter dem Einfluß von Juden - wieder preisgab. Damit verdunkelt sich sein Porträt, es gewinnt Züge der Unselbständigkeit und der Einfalt. Folglich ist es kein Wunder, wenn Cusanus nun auch harte Worte für Mohammed findet. Sie kreisen vor allem um die Frage, warum er bereit war, das einmal erreichte Christentum wieder aufzugeben. Darauf antwortet Cusanus mit einer deprimierenden Alternative: Entweder geschah es aus Unwissenheit (ignorantia); oder Mohammed verfolgte eine böse Absicht (perversitas intentionis), weil er nicht mehr Christus dienen wollte, sondern seinen eigenen Ruhm suchte (, sui ipsius gloriam quaesivit $\left.{ }^{6}\right)^{30}$.

Man sollte denken, mit solchen Sätzen sei ein definitives Urteil gesprochen. Aber Cusanus läßt es auch mit diesen düsteren Eindrücken nicht bewenden, sondern fügt seiner Einschätzung in einem späteren Teil der ,Cribratio' eine weitere Facette hinzu. Sie macht aus dem ruhmsüchtigen Ignoranten Mohammed wieder eine Figur im göttlichen Heilsplan. Das wird möglich, indem die Vorgaben der polemischen Legende, die wir gerade gehört haben, noch einmal radikal umgedeutet werden. Denn, so lautet diese dritte Überlegung ${ }^{31}$, Mohammed kannte nicht nur das Wesen des Christentums; er kannte auch seine Landsleute. Er wußte also, daß sie ohne Kultur und ohne Zivilisation, eben einfache Araber (rudes

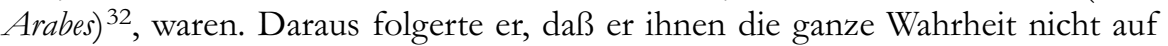
einmal zumuten durfte. Sie hätten sie ohnehin nicht verstanden und wären von ihrem Glanz eher abgeschreckt worden. Also entschloß er sich zu einem ersten pädagogischen Schritt. Er führte die Araber vom Götzendienst zum Monotheismus. Das gelang ihm mit Hilfe des Korans, der für solche Zwecke durchaus geeignet ist. Weitere Erkenntnisse, d. h. die Bekanntschaft mit dem Evangelium, konnten ja immer noch folgen. Oder, um es mit Cusanus' Worten zu sagen:

„Mohammed enthielt ihnen die Geheimnisse des Evangeliums vor (secreta evangelii occultabat) im Glauben, sie könnten zu späterer Zeit den Gebildeten eröffnet werden, wie [ja] auch das Evangelium [bei uns] anfangs vielen dunkel und unverständlich blieb und erst mit der Zeit mehr und mehr offenbar wurde.“33

30 h VIII, n. 9, 5-9.

31 Cf. h VIII, nn. 119-120; der Text steht in Zusammenhang mit dem Nachweis, daß Christus tatsächlich am Kreuz gestorben sei. Vergleichbare Interpretationsansätze finden sich allerdings schon in h VIII, n. 40 und n. 74.

32 h VIII, n. 119, 4. Hier vertritt Cusanus eine Vorstellung, die sich auch bei verschiedenen islamischen Philosophen und bei Moses Maimonides nachweisen läßt: Propheten wenden sich an ungebildete (rudes) Menschen bzw. Völker und bringen ihnen sowohl eine religiöse Botschaft als auch die Zivilisation.

33 h VIII, n. 120, 6-9. 
Die Liste der Wertungen und Urteile ließe sich ohne weiteres fortsetzen. Cusanus hat sich in der ,Cribratio“ immer wieder über die Person Mohammeds geäußert, manchmal anerkennend, manchmal abwertend. So lobt er an einer Stelle seinen Gottesglauben ${ }^{34}$ und hält ihm an anderer Stelle vor, ein notorischer Frauenheld ${ }^{35}$ gewesen zu sein. All das kann den Gesamteindruck aber nicht mehr verändern. Es bestätigt vielmehr, daß Cusanus bewußt ein zwiespältiges Bild gezeichnet hat. Für ihn war wichtig, daß Mohammed - als Prophet - am göttlichen Heilsplan mitwirkte. Aber es war ebenso wichtig, daß Mohammed als Mensch - seiner großen Aufgabe nur auf unvollkommene Weise nachkam.

Die Ambivalenz hat also System; sie gehört zum Entwurf des Cusaners. Deswegen genügt es auch nicht, wenn wir uns klarmachen, daß er die meisten Details, die er über Mohammed vortrug, bereits in seinen verschiedenen Quellen vorfand. Erst bei ihm wurde aus den Einzelheiten ein Konzept, und in diesem Konzept spiegeln sich offenbar seine philosophischen Überzeugungen. Denn vieles spricht dafür, daß das Verhältnis zwischen Mohammed und Jesus bzw. zwischen dem Islam und dem Christentum von Cusanus nach den Vorgaben der platonischen Ideenlehre definiert worden ist.

Anhaltspunkte für diese These liefert uns bereits die ,Cribratio'. Denn sie zeichnet ja eine eindrückliche Relation: hier das Christentum, gestiftet von Jesus, dem göttlichen Wort und Erlöser - dort der Islam, der von einem abtrünnigen Christen verkündet worden sei und deswegen nur als Teilhaber und unvollkommenes Abbild der Wahrheit aufgefaßt werden könne ${ }^{36}$. Die theoretische Begründung für diesen Zusammenhang finden wir in der ,Cribratio' allerdings nicht. Um sie zu erfahren, müssen wir zum anderen Text, ,De pace fidei', zurückkehren. Dort wird das Konzept der Teilhabe nämlich nicht nur angewandt, sondern auch unter der speziellen Perspektive des Dialogs zwischen den Religionen erklärt.

Den Anlaß dazu bietet eine Frage, die Cusanus dem Konferenzteilnehmer aus Griechenland in den Mund legt. Er möchte wissen, auf welcher Grundlage eigentlich die Einheit der Religionen als Ergebnis der Konferenz erreicht werden solle. Schließlich wisse man, daß jede Religionsgemeinschaft nur ihren eigenen Glauben gelten lasse und dafür sogar das Blut ihrer Märtyrer in Kauf nehme ${ }^{37}$. Die Antwort darauf gibt das göttliche Wort. Es erklärt, daß man die Religion mit der Weisheit vergleichen könne. Auch sie habe eine Vielzahl von Anhängern, die ,philosophi seu sapientiae amatores“. Aber sie alle müßten sich eingestehen, daß es nur eine Weisheit gebe, die von allen in gleicher Weise vorausgesetzt werde ${ }^{38}$.

\footnotetext{
34 h VIII, n. 99, 2-4: „Et quia Mabumetus in eo, quod deum credit creatorem omnium, etiam de ipso illa asserit necessario, quae de eius trinitate Christiani confitentur [...].“

35 h VIII, n. 44, 11-12: „[...] Mabumetus vir muliebris, lubricus, totus buius mundi et sensibilium amator $[\ldots] . “$

36 So etwa an der bereits oben (nt. 12) zitierten Stelle: „Hinc si quid pulchri, veri et clari in Alkorano reperitur, necesse est, quod sit radius lucidissimi evangelii [...].“

37 Cf. h VII, n. 10 (11, 7-10).

38 Cf. h VII, n. $10(11,11-14 ; 11,16-17 ; 11,19-21)$.
} 
Das überzeugt den Griechen. Deswegen bestätigt er umgehend: „Keiner von uns bezweifelt, daß es eine einzige Weisheit gibt [...], obwohl es viele weise Menschen gibt, die an ihr Anteil haben (cuius participatione sunt multi sapientes) [...].“39 Und so kann das göttliche Wort resümieren: „Ihr alle setzt also, obwohl ihr euch zu unterschiedlichen Religionen bekennt, in all dieser Verschiedenheit ein Einziges voraus, das ihr Weisheit nennt. “40

Von dieser Prämisse ausgehend, wurden ,De pace fidei ${ }^{‘}$ und die ,Cribratio Alkorani' geschrieben. Cusanus versucht in ihnen also, den Islam an das Christentum heranzuführen, indem er beide in ein platonisches Konzept einfügt ${ }^{41}$. Ob dieser Versuch gelungen ist, sei im Moment dahingestellt. Zunächst müssen wir uns noch einem anderen Thema zuwenden. Es betrifft die zweite Überlegung, der wir nachgehen wollten, d. h. die Frage, von welchen Grundannahmen über Gott und die Menschen Cusanus bei seiner Auseinandersetzung mit dem Islam ausging.

III.

Dazu haben wir in der ,Cribratio“ schon einige Hinweise erhalten. Denn dieses Thema kam bereits kurz zur Sprache, als sich Cusanus über die Person Mohammeds geäußert hat. In diesem Zusammenhang erklärte er, jeder Mensch wisse aus Erfahrung, daß er nach etwas strebe. Ziel dieses Strebens sei das Gute (,finis igitur desiderii bonum"), das uns anziehe und bewirke, daß sich unser Geist zu ihm hin bewege ${ }^{42}$. Dieses Gute, so die ,Cribratio“, „,nennen wir Gott, damit wir uns gegenseitig verstehen, wenn wir darüber sprechen“ („,nominamus deum, ut, dum de ipso conferimus, nos mutuo intelligamus"s $)^{43}$. Oder, wie in ,De pace fidei formuliert wird:

„[...] ein jeder strebt in dem, was er erstrebt, nur nach dem einen Guten, und das bist du. Ein jeder sucht mit seinem Verstand nur die eine Wahrheit, und die bist du. Was will ein Lebendiger anderes als leben? Was will ein Seiender anderes als sein? Da

39 h VII, n. $11(12,1-4)$.

40 h VII, n. 12 (13, 10-11): „Omnes igitur vos, etsi diversarum religionum vocemini, unum praesupponitis in omni diversitate tali, quod sapientiam nominatis." $\mathrm{Zu}$ diesem Abschnitt von ,De pace fidei“ cf. Flasch, Nikolaus von Kues (nt. 22), 345 sqq.

41 Die Ideenlehre ist im übrigen nicht das einzige platonische bzw. platonistische Konzept, das Cusanus in seinen religionsphilosophischen Überlegungen einsetzt. Daneben finden sich Hinweise auf die Anamnesis-Lehre, Spekulationen über das Verhältnis von Einheit und Vielheit (z. B. in h VII, n. 21 [20 ult.-21, 1]: „omnis autem multitudinis unitas est principium“), der Anspruch auf das Wesenswissen, die Bewertung der sinnlichen Welt als Hindernis wie als Aufstieg u. a. m.; cf. dazu im einzelnen Kremer, Die Hinführung (nt. 23), 127 sqq., sowie Flasch, Nikolaus von Kues (nt. 22), 333 sqq. u. 346 sqq.

42 h VIII, n. $5,1-7$.

43 h VIII, n. $7,8-10$. 
du das Leben schenkst und das Sein, suchen sie dich, den einen, auf unterschiedliche Weise in den verschiedenen Riten [...].“44

Cusanus vertrat also die Lehre von der natürlichen Religion, der religio con$n_{\text {nata }}{ }^{45}$. Das hätte seine muslimischen Gesprächspartner - wenn es tatsächlich zu einem Dialog gekommen wäre - durchaus interessieren können. Denn die islamischen Theologen lehren ja selbst, jeder Mensch sei von Geburt an „auf Gott hin ausgerichtet", was mit dem arabischen Wort muslim ausgedrückt wird ${ }^{46}$. Cusanus verdankte dieses Konzept aber nicht dem Islam. Er verdankte es einmal mehr - seinen philosophischen Studien. Denn auch die Annahme, daß jeder Mensch nach dem Guten strebe, konnte er bei den Philosophen nachlesen, diesmal nicht nur bei Platon, sondern auch bei Aristoteles ${ }^{47}$.

Allerdings gab Cusanus dem Konzept, das er vorfand, eine neue Wendung. Denn er betont mehrfach, daß kein einziger Mensch aus eigener Kraft, d. h. durch seine eigene intellektuelle Anstrengung, das von ihm ersehnte Gut erfassen könne. Gott sei nämlich für seine Geschöpfe unbegreifbar, „weil es zwischen dem Endlichen und dem Unendlichen keine Proportion gebe“ („cum finiti ad infinitum nulla sit proportio") ${ }^{48}$. Deswegen müßten wir sogar mit der Einsicht leben, daß sein wahrer Name ,unbekannt und unaussprechlich“ (,incognitus et ineffabilis") sei $^{49}$.

An dieser Stelle kommt die negative Theologie des Cusaners zum Tragen. Sie hat zur Folge, daß er eine tiefe Kluft zwischen Gott und den Menschen annimmt. Um sie zu überbrücken, bedarf es eines gottgewollten Vermittlers. Dieser Vermittler aber kann nach allem, was wir bislang gehört haben, nur Jesus Christus $\operatorname{sein}^{50}$. Bevor Cusanus diese Schlußfolgerung zieht, macht er indessen noch eine Konzession. Er spricht davon, daß Gott zunächst versucht habe, die Menschen auf anderen Wegen zum Heil zu führen. Dabei fallen wieder Äußerungen, die für uns interessant sind, weil sie noch einmal auf die Stellung der Propheten eingehen.

Jetzt erfahren wir nämlich, daß Gott mehrmals ,verschiedene Könige und Seher, die Propheten genannt werden“ (,diversos reges et videntes, qui prophetae dicuntur $\left.^{\prime \prime}\right)$, zu den Menschen gesandt habe. Sie traten jeweils an die Spitze eines Volkes, erließen Gesetze und unterwiesen das ,einfache Volk ${ }^{\star}$ (,rudem populum ${ }^{\varsigma}$ )

\footnotetext{
44 h VII, n. 5 (6, 11-15).

45 Dazu ausführlich Euler, Nikolaus von Kues als Wegbereiter (nt. 2), 213-216.

46 Zu den koranischen Wurzeln dieser Vorstellung cf. T. Nagel, Der Koran. Einführung - Texte Erläuterungen, München 1983, ${ }^{3} 1998,252-254$.

47 Vor allem in der berühmten Eröffnung zur ,Nikomachischen Ethik'; zum Grundbegriff des Strebens, der dort eingeführt wird, cf. O. Höffe, Aristoteles, München 1996, ${ }^{2} 1999,202-$ 205.

48 h VII, n. $5(7,1-2)$.

49 h VII, n. 5 (6 ult.); zum Motiv der Verborgenheit Gottes ausführlich Euler, Cusanus' Verständnis (nt. 2), 137 sqq., sowie Nikolaus von Kues als Wegbereiter (nt. 2), 217 sqq.

50 Cf. ibid., 220 sqq.
} 
in den religiösen Fragen, zu denen es von sich aus keinen Zugang hatte ${ }^{51}$. All das geschah nach Gottes Plan, aber das Ergebnis war niemals vollkommen. Denn es kam immer wieder zu Fehlentwicklungen, unter denen Cusanus drei hervorhebt. Erstens: Die Menschen nahmen die Propheten zu ernst. Sie trennten nicht zwischen Gott und seinen Gesandten. So kam es, daß sie die religiösen Gesetze so befolgten, „als hättest du selbst als allerhöchster König sie ihnen von Angesicht zu Angesicht diktiert, weil sie dich, nicht jene (d.h. die Propheten) darin zu hören glaubten“ ${ }^{52}$. Zweitens: Auch die Propheten begingen Fehler, denn sie waren nun einmal Menschen. Das hatte zur Folge, daß sie Gott eigentlich gar nicht erfassen konnten und außerstande waren, seine Botschaft den anderen Menschen zu erklären ${ }^{53}$. Drittens: Man muß in dieser Welt immer mit den Einflüssen des Bösen rechnen. Er findet stets einen Weg, die Menschen zu verführen und zu überlisten. Das bringt Cusanus zum Ausdruck, indem er Gott in ,De pace fidei‘ sagen läßt:

„In mühevoller Fürsorge habe ich versucht, den Umherirrenden durch verschiedene Propheten, die im Vergleich zu ihren Zeitgenossen wie Sehende unter Blinden waren, auf den rechten Weg zurückrufen zu lassen. Doch all diese Propheten waren nicht stark genug, den Herrscher der Dummheit (principem ignorantiae) zu besiegen. " 54

Die Konsequenz, die Cusanus aus diesen Überlegungen gezogen hat, ist uns inzwischen bekannt. Er sah angesichts der genannten Probleme nur eine Lösung: Gott mußte seinen eigenen Sohn in die Welt schicken, um das Böse zu überwinden und die Menschen wieder auf den rechten Weg zu führen ${ }^{55}$. Seither ist die Wahrheit bekannt; man kann sie im Christentum finden. Das bewirkte zwar nicht die Auflösung der anderen Religionen. Aber es führte immerhin dazu, daß sich jetzt sämtliche Glaubensgemeinschaften an der christlichen Botschaft orientieren können.

Man muß fairerweise hinzufügen, daß Cusanus auch andere Versuche unternommen hat, um die Vielfalt der Religionen zu erklären. Einmal wird die Verborgenheit Gottes als Grund dafür genannt, einmal die begrenzte Fassungskraft der Menschen ${ }^{56}$. Außerdem spielt er an einer Stelle sogar mit der Möglichkeit, daß in der Vielfalt der Glaubensformen ein Gewinn liegen könnte. So kann man jedenfalls zwei Sätze, die der Erzengel in der Einleitung zu ,De pace fidei` an Gott richtet, verstehen. Sie lauten: „Die verschiedenen Riten zu vereinheitlichen

51 Cf. h VII, n. $4(5,18-6,1)$.

52 h VII, n. 4 (6, 1-3): ,[...] ac si tu ipse Rex regum facie ad faciem eis locutus fuisses [...], non eos sed te in ipsis audire credentes."

53 h VIII, n. 8, 7-12: „Unde si nec Moyses nec Mabumetus umquam, dum in hoc mundo essent, saepe dictum bonum viderunt - deum enim nemo vidit umquam -, quomodo tunc aliis iter ad ipsum pandere potuerunt? Esto autem, quod sermones aliquos eis immissos publicassent, qui figurabant seu significabant deum et viam ad ipsum, tamen ipsi illos exponere non potuissent neque alius homo."

54 h VII, n. 7 (8, 9-13).

55 Cf. h VII, n. 7 (8, 13-17).

56 Beispiele für beides bei Euler, Cusanus' Verständnis (nt. 2), 137 sq., und id., Nikolaus von Kues als Wegbereiter (nt. 2), 216 sqq. 
ist weder möglich noch wünschenswert. Denn dadurch kümmert sich ja jedes Volk besonders hingebungsvoll um seine eigenen Bräuche, weil es denkt, gerade diese seien dir die liebsten." 57

Leider wird die Engelsrede mit den Worten fortgesetzt: „Doch wie du ein Einziger bist, soll es auch nur eine einzige Religion geben [...]. "58 Damit sind wir sehr schnell wieder bei den grundsätzlichen Überzeugungen des Cusaners angekommen. Sie erweisen sich als stabil. Das belegen sowohl seine Bemerkungen über Mohammed als auch seine Äußerungen über die religiöse Natur des Menschen. Denn sie haben jeweils gezeigt, daß er bei seiner Einschätzung der verschiedenen Religionen von einer eindeutigen Rangordnung, genauer gesagt: von der Relation zwischen dem einen vollkommenen Urbild und den zahlreichen, nur partiell gelungenen Abbildern ausgeht.

Wir können also resümieren, daß Cusanus an seiner platonisierenden Auslegung der Religionen festhielt. Damit ist aber nicht gesagt, daß diese Auslegungsordnung für den Dialog zwischen den Religionen förderlich gewesen sei. Im Gegenteil: Der Gesamteindruck, den uns ,De pace fidei' und die ,Cribratio Alkorani‘ vermittelt haben, war eher zwiespältig. Denn wir konnten beobachten, daß der Rückgriff auf die Philosophie vor allem zu zwei Konsequenzen geführt hat. Einerseits erhielt Cusanus durch die Ideenlehre die Möglichkeit, den Islam und das Christentum - über das Konzept der Teilhabe - systematisch aneinander zu binden. Das kann man durchaus als einen Gewinn betrachten, vor allem dann, wenn man die polarisierenden Äußerungen zahlreicher älterer und jüngerer Autoren zum Islam liest. Andererseits hatte dasselbe Konzept aber auch einen gegenteiligen Effekt. Denn es führte dazu, daß die Hierarchie zwischen den beiden Religionen akzentuiert wurde. Sie beruht bei Cusanus nicht mehr auf dem schlichten Gefühl der Überlegenheit oder auf simpler Polemik, sondern hat eine systematische Begründung erhalten, die als bewiesen, notwendig und unumstößlich gilt.

Hinzu kommt ein letztes Problem. Gemeint ist die Frage, was Cusanus - als er von der platonischen Ideenlehre ausging - als Absolutum, d. h. als die Idee des Guten voraussetzte. Hier sind seine Äußerungen nicht immer eindeutig. Denn zu Beginn seiner Überlegungen wird allein Gott als das vollkommene Gute bezeichnet. Später im Text gewinnt man dagegen den Eindruck, daß auch die christliche Religion in den Rang des Absoluten aufsteigt. Das aber ist entscheidend. Denn daran wird die Möglichkeit, ob es zu einem Dialog kommen kann, gemessen. Sie dürfte verstellt sein, wenn eine Seite ihre Religion als den alleinigen und vollkommenen Maßstab betrachtet - gleichgültig, ob dieser Anspruch mit banalen Argumenten oder durch die platonische Philosophie begründet wird.

57 h VII, n. 6 (7, 11-14): „Quod si forte haec differentia rituum tolli non poterit aut non expedit, ut diversitas sit devotionis adauctio, quando quaelibet regio suis ceremoniis quasi tibi regi gratioribus vigilantiorem operam impendet."

58 h VII, n. 6 (7, 14-15): ,saltem ut sicut tu unus es, una sit religio [...].“ 
Daß es im Mittelalter durchaus christliche Denker gab, die über Cusanus' Position hinausgingen und bereit waren, fremde Religionen (namentlich die islamische) in ihrer Eigenart und Andersheit zu respektieren, zeigt das Beispiel des ägyptischen Gelehrten al-Ṣafī ibn al- 'Assāl (gest. um 1265). Er war einer der maßgeblichen Autoren der koptischen Kirche und verfaßte zahlreiche Werke zur Dogmatik, zur Pastoraltheologie und zum kirchlichen Recht ${ }^{59}$. Zu diesen Werken zählt eine Schrift mit dem Titel, Die Wahrheiten bezüglich der Antwort auf die guten Ratschläge' (Al-Ṣahā̄ iḥ fī ğawāb al-nașā̉ih). Sie gehört eigentlich in das Genre der Apologetik, denn der Text ist als Antwort auf die antichristliche Polemik des 'Alī ibn Rabbān al-Ṭabarī (gest. um 855) konzipiert ${ }^{60}$. Gleichwohl nutż al-Ṣafī seine Darstellung, um religionsphilosophische Betrachtungen anzustellen. Das zeigt sich vor allem in einem kurzen Textabschnitt, den Khalil Samir separat ediert, ins Französische übersetzt und kommentiert hat ${ }^{61}$. Dort deutet der koptische Gelehrte an, daß die Vielheit/Vielfalt der Religionen nicht nur als Problem, sondern auch als Gewinn betrachtet werden könne. Das wird auf drei Ebenen begründet: im Hinblick auf Gott, auf den einzelnen Menschen und auf die verschiedenen Glaubensgemeinschaften, die sich in der Welt konstituiert haben.

Wörtlich heißt es dazu:

„(41) Le Sage Très-Haut a sans doute en ceci un plan mystérieux. (42) Par exemple de laisser une minorité se séparer, dans diverses opinions, de l'opinion de la majorité, (43) afin qu'il n'y ait pas un [seul] aspect, parmi tous ceux que l'on peut penser appartenir aux attributs de la perfection, qui ne soit attribué à Dieu; (44) ou afin que se manifeste l'opinion la meilleure, du milieu de toutes les opinions; (45) ou afin que l'on parvienne, grâce à l'effort (iğtihäd) causé par la concurrence, à ce à quoi on ne pourrait parvenir grâce à la science et à l'action sans concurrence; (46) ou encore afin que l'on aboutisse, grâce à la solidarité existant à l'intérieur de chaque communauté, à repousser les nuisances qui s'attaquent à ses membres (47) (qu'elles soient causées par la malchance, ou la mauvaise planification, ou l'injustice des autres), (48) ce qui n'aurait pas eu lieu s'ils appartenaient tous à une seule religion. (49) Et c'est pourquoi cette solidarité est plus forte à l'intérieur d'une petite communauté qu'à l'intérieur d'une grande." 62

59 Dazu G. Graf, Geschichte der christlichen arabischen Literatur. II: Die Schriftsteller bis zur Mitte des 15. Jahrhunderts (Studi e Testi 133), Città del Vaticano 1947, 388-403.

${ }^{60}$ Cf. Kh. Samir, La réponse d'al-Ṣafí Ibn al-'Assāl à la Réfutation des chrétiens de 'Alī al-Ṭabarī, in: Parole de l'Orient 11 (1983), $281-328$.

61 L'accord des religions monothéistes entre elles selon al-Ṣafī Ibn al-`Assāl, in: Proche-Orient Chrétien 36 (1986), 206-229.

62 L'accord (nt. 61); arab. Text 221; frz. Übersetzung 220; Kapiteleinteilung und Übersetzung nach Samir. 